\title{
The Modified Microscope Drape Technique: A Self-Contained Surgical Working Space in Endoscopic Sinus Surgery and Transoral Robotic Surgery in COVID-19 Era.
}

\author{
Naseem Ghazali ${ }^{1}$ and Anu Daudia ${ }^{2}$ \\ ${ }^{1}$ East Lancashire Hospitals NHS Trust \\ ${ }^{2}$ Royal Blackburn Hospital
}

June 18, 2020

\begin{abstract}
The microscope drape has been modified recently to provide an additional barrier to airborne transmissions of the SARS-CoV-2 virus. We describe the application of the modified microscope drapes in elective endoscopic sinus surgery and transoral robotic surgery as a standard operating protocol.
\end{abstract}

The Coronavirus disease (COVID-19) caused by the virus Severe acute respiratory syndrome coronavirus 2 (SARS-CoV-2) has significantly impacted upon otolaryngology, oral and maxillofacial, and head and neck surgery due to the risk of airborne transmission of SARS-CoV-2 infection ${ }^{1,2}$. The aerosols generated during medical procedures (AGMP) remain active over time once airborne, and can spread over distances by air currents, posing immediate risk to operation room (OR) staff. Recognising that not all medical procedures can be deferred, clinical recommendations regarding case selection, mandatory preoperative patient selfisolation and COVID testing, perioperative optimisation of infection control and OR management, and postoperative care has allowed resumption of elective surgical activity ${ }^{1,2}$.

The concept of an aerosol box was introduced in March 2020 to provide an additional barrier during AGMP, particularly during intubation ${ }^{3}$. However, this three-sided rigid box does not completely enclose the highrisk area nor does it contain the aerosols generated during the procedure. Rokade et al [2020] recently modified the microscope drape to create a completely enclosed working area during endoscopic sinus surgery (ESS) ${ }^{4}$. The drape is used to cover the patient's head and torso and is tented up by horizontal bar. The three ocular arms of the drape were used to provide access for the surgeon's arms and camera, while the lens cover was used to pass instruments into the tent.

In our hospital, modifications to Rokade et al's original description were made to enhance the workflow encountered during ESS. A Mayo table was placed at the head end to provide additional work top for instruments. An additional horizontal bar was placed between the Mayo table and the original horizontal bar. When draped, the three ocular arms were positioned over the Mayo table, nose and chest, respectively, allowing for greater freedom of movement during surgery. One of the ocular arms provided access for the scrub nurse's arm into the working space to assist the surgeon with instrumentation. Two additional access openings into the drape were made for the surgeon's arms. A larger sized microscope drape (Invisihield ${ }^{\mathrm{TM}}$ ICE5320, Medline International) was chosen to ensure that an adequate tent height was achieved to accommodate the working space which includes the Mayo table, while maintaining unrestricted movement of the surgeon's and scrub nurse's arms during surgery [Figure 1A and 1B].

Our hospital also uses a version of the modified microscope drape for transoral robotic surgery (TORS). 
Ghazali et al [2020] recently described a self-contained chamber enclosing the patient and the aerosols generated during TORS by modifying the microscope drape ${ }^{5}$. Once the drape was placed around the patient and secured at the mid-abdomen, three puncture holes were made in the drape to allow placement of the three robotic cannulas for the two endowrist instruments and the da Vinci 3D endoscope at the level of the patient's mouth. The drape was secured around the cannulas using tape. The robot surgeon assistant gains access into the self-contained chamber through openings made in the two outer ocular arms conveniently found at the head end of the patient. Aerosols generated by energy instrumentation in the pharynx/upper airways were removed from the self-contained chamber by applying continuous high vacuum suction. This suction tube was attached to the Luer Lock of the robotic cannula, and a connection to a laparoscopic HEFA filter removes airborne particles including viruses throughout the procedure [Figure 1C]. Additional suction is customarily applied in the nasopharynx. Finally, careful 'doffing' of the microscope drape at the end of the procedure prevents dispersion of any trapped aerosols within the drape.

Currently, the modified microscope drape system is a standard practice for both ESS and TORS at our hospital, and preliminary work to evaluate its efficacy is being undertaken.

Conflict of interest: None

\section{References:}

1. Lammers MJW, Lea J, Westerberg BD. Guidance for otolaryngology health care workers performing aerosol generating medical procedures during the COVID-19 pandemic. J Otolaryngol Head Neck Surg . 2020;49(1):36. Published 2020 Jun 3. doi:10.1186/s40463-020-00429-2

2. Kowalski LP, Sanabria A, Ridge JA, et al. COVID-19 pandemic: Effects and evidence-based recommendations for otolaryngology and head and neck surgery practice. Head Neck . 2020;42(6):1259-1267. doi:10.1002/hed.26164

3. Lai HY, Design A (2020) Aerosol box-design. [online] Sites.-google.com. Available at: https://sites.google.com/view/aerosol box/design. (Accessed 13 June 2020)

4. Rokade A, Burgess A, Koch HI, Tsagkovitis, Ioannidis D. FESS in the COVID Era. The microscope drape methd to reduce aerosolization. Available at: https://www.entuk.org/fess-covid0era-microscopedrape-method-reduce-aerosolization. (Accessed 13 June 2020)

5. Ghazali N, Attardo A, Markose G. Modification of the Microscope Drape to Provide a Closed Surgical Field in Transoral Robotic Surgery. Accepted by British Journal of Oral \& Maxillofacial SurgeryBJOMS-D-20-00765, 16 June 2020. 

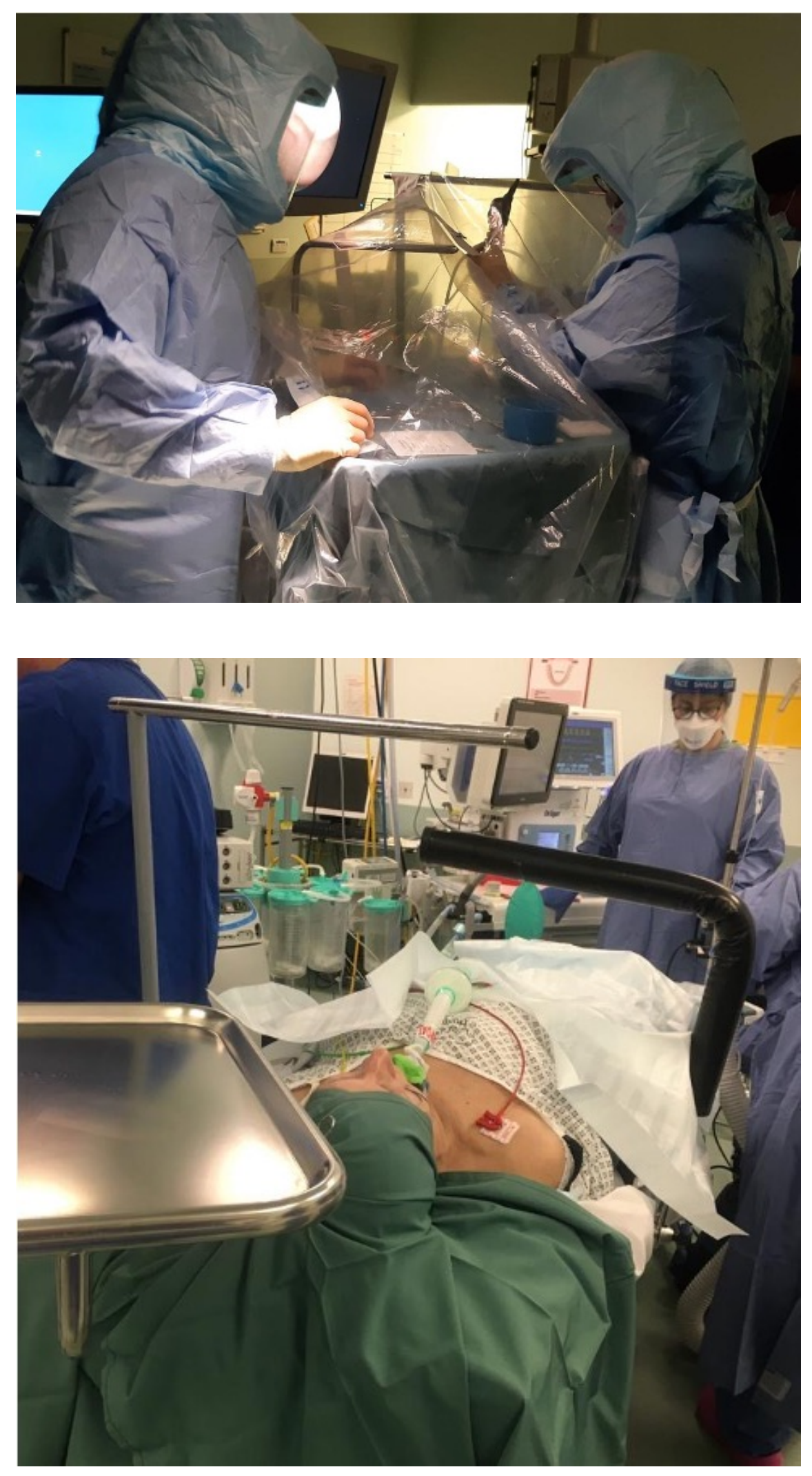


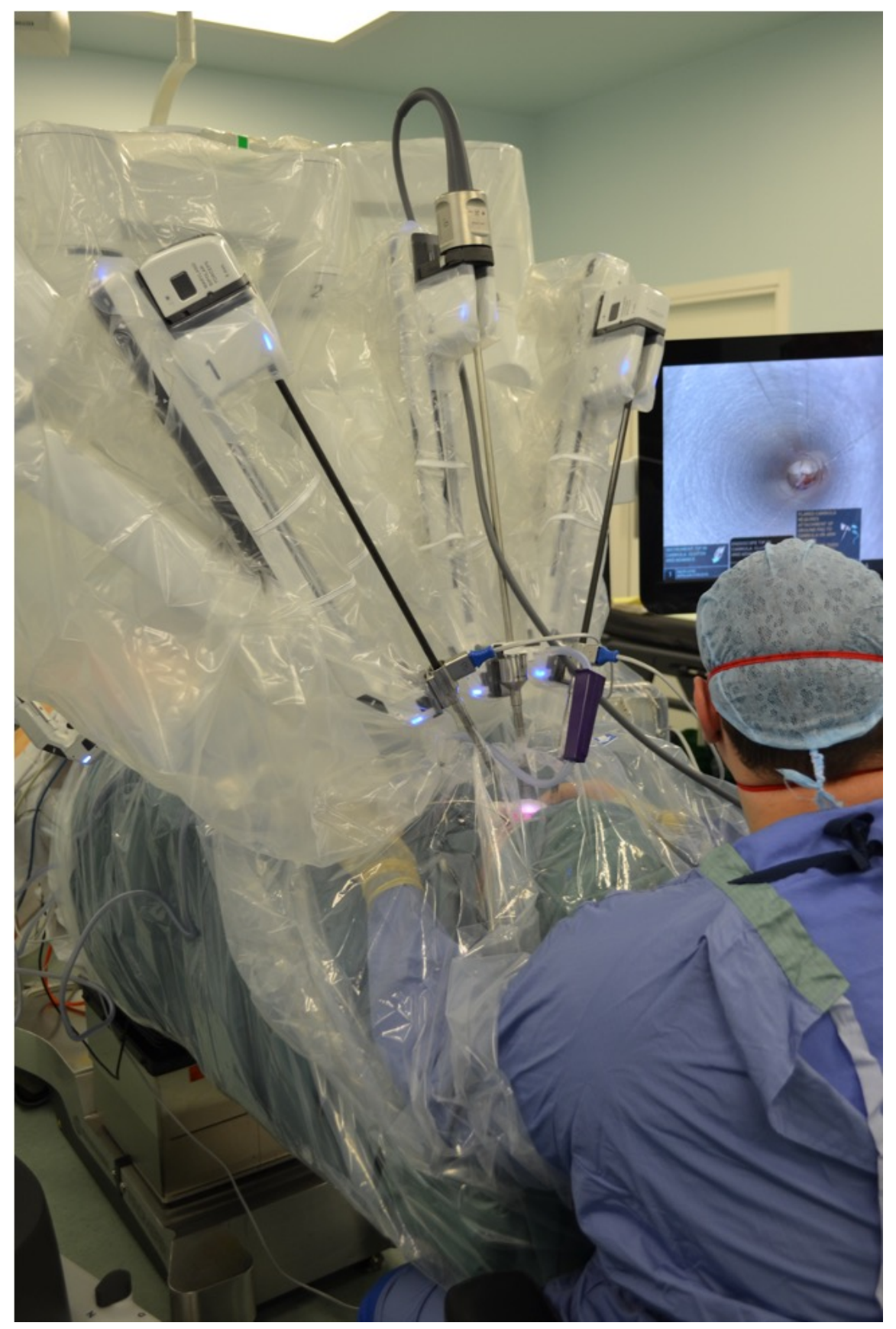

